\title{
DEGENERATE BAND EDGES IN PERIODIC QUANTUM GRAPHS
}

\author{
GREGORY BERKOLAIKO AND MINH KHA
}

\begin{abstract}
Edges of bands of continuous spectrum of periodic structures arise as maxima and minima of the dispersion relation of their Floquet-Bloch transform. It is often assumed that the extrema generating the band edges are non-degenerate.

This paper constructs a family of examples of $\mathbb{Z}^{3}$-periodic quantum graphs where the non-degeneracy assumption fails: the maximum of the first band is achieved along an algebraic curve of co-dimension 2 . The example is robust with respect to perturbations of edge lengths, vertex conditions and edge potentials. The simple idea behind the construction allows generalizations to more complicated graphs and lattice dimensions. The curves along which extrema are achieved have a natural interpretation as moduli spaces of planar polygons.
\end{abstract}

\section{INTRODUCTION}

Periodic media play a prominent role in many fields including mathematical physics and material sciences. A classical instance is the study of crystals, one of the most stable form of all solids that can be found throughout nature. In a perfectly ordered crystal, the atoms are placed in a periodic order and this order is responsible for many properties particular to this material. On the mathematical level, the stationary Schrödinger operator $-\Delta+V$ with a periodic potential $V$ is used to describe the one-electron model of solid state physics [1]; here $V$ represents the field created by the lattice of ions in the crystal. The resulting differential operator with periodic coefficients has been studied intensively in mathematics and physics literature for almost a century. A standard technique in spectral analysis of periodic operators is called the Floquet-Bloch theory (see e.g., [26, 27]). This technique is applicable not only to the above model example of periodic Schrödinger operators on Euclidean space, but also to a wide variety of elliptic periodic equations on manifolds and branching structures (graphs). Periodic elliptic operators of mathematical physics as well as their periodic elliptic counterparts on manifolds and quantum graphs do share an important feature of their spectra: the so-called band-gap structure (see e.g., 10, 24, 26, 27]). Namely, the spectrum of a periodic elliptic operator can be represented in a natural way as the union of finite closed intervals, called spectral bands, and sometimes they may leave open intervals between them, called spectral gaps. An endpoint of a spectral gap is called a gap edge. For each spectral band, there is also a corresponding band function whose image is exactly that spectral band. The set consisting of all graphs of band functions is called the dispersion relation. The analytical and geometrical properties of dispersion relations encode significant information about the spectral features of 
the operator 1 Hence studying structural properties of the dispersion relation may reveal interesting results for periodic differential operators. A well-known and widely believed conjecture in physics literature says that generically (with respect to perturbations of the coefficients of the operator) the extrema are attained by a single band of the dispersion relation, are isolated, and have non-degenerate Hessian. The non-degeneracy of extrema at the edges of the spectrum is often assumed to establish many important results such as finding asymptotics of Green's functions of a periodic elliptic operator near and at its gap edge [20,21,30], homogenization [12 14], or counting dimensions of spaces of solutions with polynomial growth [28, 29], just to name a few.

In the continuous situation, the generic simplicity of spectral gap edges was obtained in $[23]$. The well-known result in $[22$ established the validity of the full conjecture for the bottom of the spectrum of a periodic Schrödinger operator in Euclidean spaces, however the full conjecture still remains unproven for internal edges. It is worth mentioning that in the two dimensional situation, a "variable period" version of the non-degeneracy conjecture was found in [34] and the isolated nature of extrema for a wide class of $\mathbb{Z}^{2}$-periodic elliptic operators was recently established in [18]. In the discrete graph situation, the statement of the conjecture fails for periodic Schrödinger operators on a diatomic lattice (see [18]). However, in the example of [18] there are only 2 free parameters to perturb the operator with and therefore the degeneracy may be attributed to the paucity of available perturbations. To investigate this question further, [15] considered a wider class of $\mathbb{Z}^{2}$-periodic discrete graphs and it was found that the set of parameters of vertex and edge weights for which the dispersion relation of the discrete Laplace-Beltrami operator has a degenerate extremum is a semi-algebraic subset of co-dimension 1 in the space of all parameters. These examples show that the non-degeneracy of gap edges is a delicate issue even in the discrete setting.

In this paper, we propose two examples of periodic metric (or "quantum") graphs whose Schrödinger operator dispersion relation has a degenerate band edge. Remarkably, this band edge remains degenerate under a continuum of perturbations: one may vary edge lengths, vertex coupling constants and the edge potentials. Our examples can be considered quantum-graph versions of the counterexample in [18], and they clearly show that the main reason for the degeneracy is not the small number of perturbation degrees of freedom, but rather the drastic effect a suitably chosen rank-1 perturbation has on the topology of the graph.

\section{The MAIN RESULT}

We now introduce the quantum graph of our main theorem and formulate the result. The description of principal notions used in the main theorem, such as quantum graphs, covers and periodicity, and the Floquet-Bloch transform, are deferred to Sections 3.1, and 3.2 correspondingly. Expanded versions of these descriptions are available in several sources, such as [6, 10, 27, 35.

\footnotetext{
${ }^{1}$ These features are also called "threshold effects" 13 whenever they depend only on the infinitesimal structure (e.g., a finite number of Taylor coefficients) of the dispersion relation at the spectral edges.
} 
We will in fact describe two variants of our graph, $X_{1}$ and $X_{2}$; the main theorem will apply equally to both. We start by describing one layer of the graph, which looks like planar hexagonal lattice shown in Figure 1. It has vertices of two types, type $A$ and type $B$ denoted by red filled and blue empty circles correspondingly. The graph $X_{1}$ will have $\delta$-type conditions at vertices $A$ and $B$, with real coupling constants $\gamma_{A}$ and $\gamma_{B}, \gamma_{A} \neq \gamma_{B}$. The graph $X_{2}$ will have only Neumann-Kirchhoff (NK) conditions but the vertices of type $A$ are decorated by attaching a "tail", i.e. an edge leading to a vertex of degree one, shown as a smaller black circle in Figure 1(right). Either version is a $\mathbb{Z}^{2}$-periodic graph in $\mathbb{R}^{2}$ and its period lattice is generated by the two brown dashed vectors. The edges of the same color (parallel edges) are related by $\mathbb{Z}^{2}$-shifts. They are assumed to have the same length and to have the same potential (if any) placed on them.
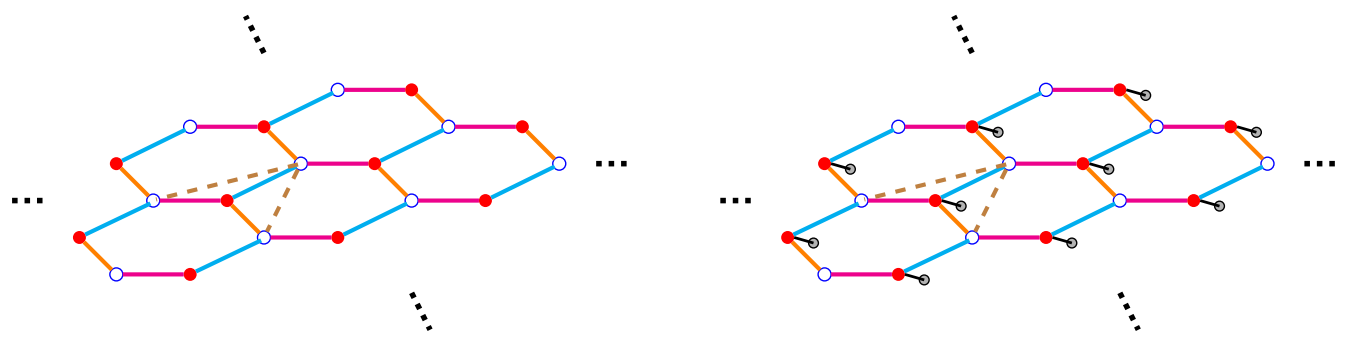

FiguRE 1. Two layers of graphs $X_{1}$ (left) and $X_{2}$ (right) respectively. These layers are $\mathbb{Z}^{2}$-periodic with respect to the Bravais lattice generated by the two brown dashed. The only difference between these two layers is the extra black tails added in the right layer.

The $\mathbb{Z}^{3}$-periodic graphs $X_{1}$ and $X_{2}$ are obtained by stacking the corresponding layers infinitely many times in both directions of the height axis, see Figure 2. The layers are connected in a periodic fashion by edges (shown in green) between vertices of type B in a lower level and vertices of type A in the upper level. Roughly speaking, one may think of the result as an infinite sheeted cover of the layers in Figure 1. In particular, $X_{1}$ is a 3-dimensional topological diamond lattice, see [35]. In Figure 3 we sketch a choice of the fundamental domain of the graph $X_{1}$ with respect to the $\mathbb{Z}^{3}$-periodic lattice.

The graphs $X_{1}$ and $X_{2}$ we defined above are actually the maximal abelian covers of finite graphs (see e.g., [2,35] for more details on maximal abelian covers of graphs). Taking the quotient of $\bar{X}$ with respect to the periodic lattice we obtain the respective graphs in Figure 4. The graph $\Gamma_{1}=X_{1} / \mathbb{Z}^{3}$ has two vertices, $A$ and $B$, which are the images of the vertices of type $A$ and $B$ in $X_{1}$ under the canonical covering map from $X_{1}$ to $\Gamma_{1}$. The four edges of $\Gamma_{1}$ are the images of the sets of parallel edges in $X_{1}$. The graph $\Gamma_{2}=X_{2} / \mathbb{Z}^{3}$ has three vertices and five edges. For either graph $\Gamma$, the first integral homology group is $H_{1}(\Gamma, \mathbb{Z}) \cong \mathbb{Z}^{3}$. We will be using notation $X$ when a statement applies equally to both $X_{1}$ and $X_{2}$; similarly we use $\Gamma$ to refer to both graphs $\Gamma_{1}$ and $\Gamma_{2}$.

The graphs $X$ are metric graphs: each edge $e$ in $X$ is identified with the interval $[0, \ell(e)]$, where $\ell(e)$ is the length of the edge $e$. The lengths of edges related by a 


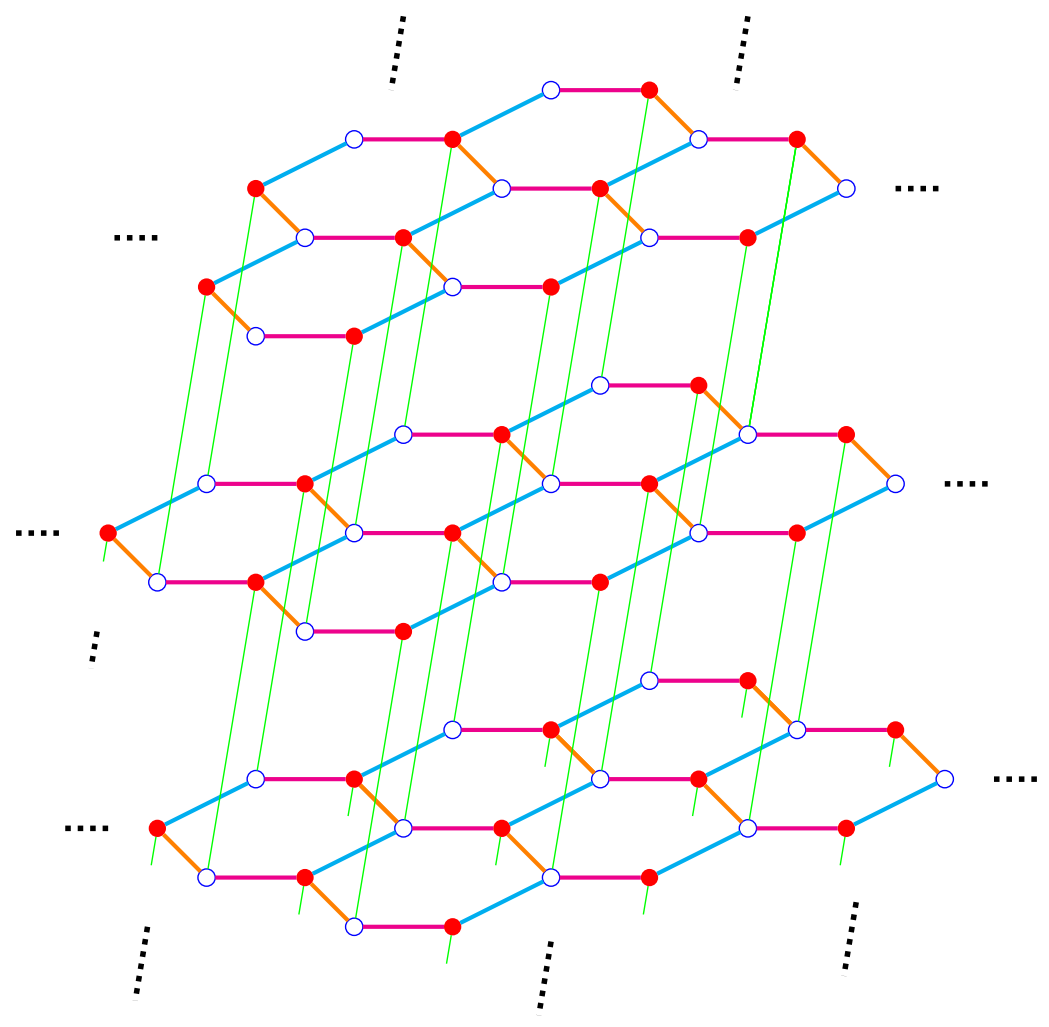

FiguRE 2. The graph $X_{1}$ is generated by stacking together infinitely many copies of the layer graph along the height axis. A layer is connected to the next layer by certain green edges. To get the graph $X_{2}$, one just simply adds black tails at the red filled vertices of $X_{1}$.

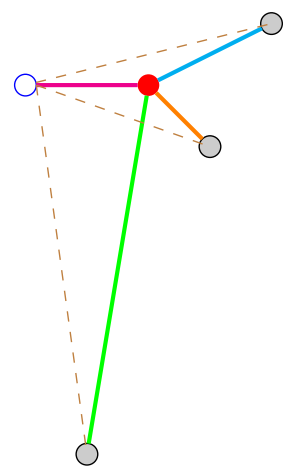

Figure 3. A fundamental domain for the graph $X_{1}$. Here the three gray vertices are not included in the fundamental domain. The graph $X_{1}$ can be obtained by shifting this fundamental domain along the three dashed directions, which are its periods.

periodic shift (i.e. belonging to the same $\mathbb{Z}^{3}$-equivalence class or having the same color) are the same. We denote by $\ell_{j}, j \in\{1, \ldots, 4\}$ the distinct lengths of edges 

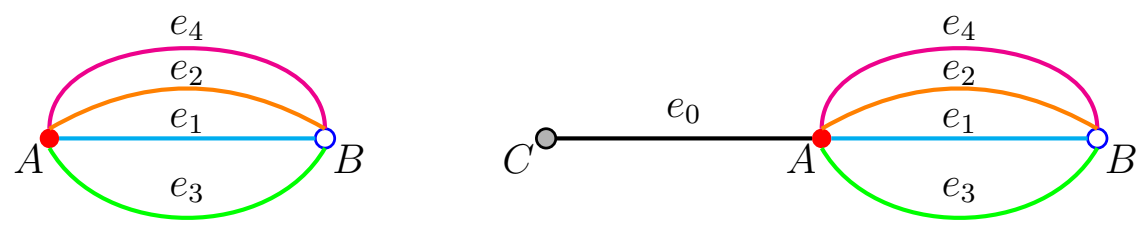

Figure 4. The graph $\Gamma_{1}$ (left) and the graph $\Gamma_{2}$ (right). In both graphs, the vertices $A, B$ correspond to red-filled and blue-empty type vertices in $X$, while the vertex $C$ corresponds to the decorated vertices in the black tails in $X_{2}$. Here $e_{0}$ is the line $C A$ and $e_{j}, 1 \leq j \leq 4$ are the corresponding edges between the two vertices $A$ and $B$.

in the graph $X_{1}$; the graph $X_{2}$ has an additional length - the length of the tail - which we denote by $\ell_{0}$. This metric information on $X$ can be viewed as a pullback of the metric on $\Gamma$ via the covering map $\pi: X \rightarrow \Gamma$. Notice that unlike the periodic realization of graphene and its multi-layer variants, we do not assume that each hexagon in the layer graph is regular, i.e. the lengths of edges with distinct colors may be different.

On the edges of the graph $X$ we consider the Laplacian $-\Delta_{X}=-\frac{d^{2}}{d x^{2}}$ or, more generally, the Schrödinger operator $-\Delta_{X}+q_{e}(x)$ with piecewise continuous potential $q_{e}(x)$. The potential is assumed to be the same on the edges of the same equivalence class (color), taking into account the edge's orientation. This ensures the potential is $\mathbb{Z}^{3}$-periodic like the rest of the graph; we do not impose any other symmetry conditions on $q_{e}$. Regularity of the potential also plays no role in our examples, the same results can be extended to $L^{1}$ potentials with minor modifications.

At every vertex of the graph $X_{2}$, we impose the standard Neumann-Kirchhoff boundary condition; we impose $\delta$-type conditions with distinct coupling constants $\gamma_{A}$ and $\gamma_{B}$ (one of them may be zero) on the corresponding vertices of the graph $X_{1}$. For the precise definition of vertex conditions, the reader is referred to Section 3.1. The graphs $X$ are non-compact, $\mathbb{Z}^{3}$-periodic quantum graphs. According to the FloquetBloch theory, the spectrum of the operator $-\Delta_{X}$ is the union of the ranges of the band functions $\lambda_{j}=\lambda_{j}(k), j \geq 1$, where the quasimomentum $\mathbf{k}$ ranges over the torus $\mathbb{T}^{3}:=(\mathbb{R} / 2 \pi \mathbb{Z})^{3}=(-\pi, \pi]^{3}$ and

$$
\lambda_{1}(\mathbf{k}) \leq \lambda_{2}(\mathbf{k}) \leq \cdots \quad \text { for any } \mathbf{k} \in \mathbb{T}^{3} .
$$

Now we state our main result.

Theorem 2.1. (a) The spectrum of the operator $-\Delta_{X}$ has an open gap between the first and the second band functions, i.e.

$$
\max _{\mathbf{k}} \lambda_{1}(\mathbf{k})<\min _{\mathbf{k}} \lambda_{2}(\mathbf{k})
$$

(b) If the lengths $\ell_{j}(1 \leq j \leq 4)$ are approximately equal, then there exists a non-trivial one-dimensional algebraic curve $\mu$ in $\mathbb{T}^{3}$ such that $\lambda_{1}$ attains its maximum value on $\mu$. Consequently, there exists a degenerate band edge in the spectrum of $-\Delta_{X}$. 
(c) The degenerate band edge in the spectrum is persistent under a small perturbation of edge lengths, vertex coupling constants or edge potentials.

Theorem 2.1 will be proved in Section 4 after reviewing relevant definitions and tools in Section 3. It will become clear during the proof that the phenomenon described in the Theorem is very robust. Informally speaking, the extremum responsible for a band edge is frequently degenerate for any graph where removing a single vertex (but not the edges incident to it) reduces the rank of the fundamental group by 3 or more. In particular, the condition on the edge lengths in part (b) of the Theorem serves only to insure a degenerate band edge particularly for the first band. For almost all choices of edge lengths one can show that a finite proportion of bands will have degenerate edges:.$^{2}$

The decorations introduced at vertices $A$ and $B$ ( $\delta$-type conditions in $X_{1}$ and the tail edge in $X_{2}$ ) serve to break symmetry in the periodic graph and thus create a band gap. If the symmetry is not broken, one would expect the bands to touch along the curve $\mu$; see [7] for related results. Finally, the topology of the degeneracy submanifold $\mu$ may be non-trivial in the higher-dimensional analogues of our example. We touch upon it in in Section 5 .

\section{Some PRELIminaries AND NOtATIONS}

3.1. Quantum graphs and vertex conditions. In this section we recall some notations and basic notions of quantum graphs; for more details the reader is encouraged to consult [10,33]. Consider a graph $\mathcal{G}=(\mathcal{V}, \mathcal{E})$ where $\mathcal{V}$ and $\mathcal{E}$ are the sets of vertices and edges of $\mathcal{G}$, respectively. For each vertex $v \in \mathcal{V}$, let $\mathcal{E}_{v}$ be the set of edges $e$ incident to the vertex $v$. The degree $d_{v}$ of the vertex $v$ is the cardinality of the set $\mathcal{E}_{v}$. The graph $\mathcal{G}$ is a metric graph if each edge $e$ of the graph is give a length, $\ell_{e}$ and can thus be identified with the interval $\left[0, \ell_{e}\right]$. A function $f$ on the graph $\mathcal{G}$ is henceforth a collection of functions $\left\{f_{e}\right\}_{e \in \mathcal{E}}$, each defined on the corresponding interval.

Let us denote by $L^{2}(\mathcal{G})$ (correspondingly $H^{2}(\mathcal{G})$ ) the space of functions on the graph $\mathcal{G}$ such that on each edge $e$ in $\mathcal{E}, f_{e}$ belongs to $L^{2}(e)$ (corresp. $H^{2}(e)$ ) and, moreover,

$$
\sum_{e \in \mathcal{E}}\|f\|_{L^{2}(e)}^{2}<\infty \quad\left(\text { corresp. } \sum_{e \in \mathcal{E}}\|f\|_{H^{2}(e)}^{2}<\infty\right) .
$$

$\mathcal{G}$ is called a quantum graph if it is a metric graph equipped with a self-adjoint differential operator $\mathcal{H}$ of the Schrödinger type acting in $L^{2}(\mathcal{G})$. We will take $\mathcal{H}$ to act as $-\Delta_{\mathcal{G}}+q_{e}(x)$ on the edge $e$, where $q_{e}$ are assumed to be piecewise continuous. The domain of the operator will be the Sobolev space $H^{2}(\mathcal{G})$ further restricted by a set of vertex conditions which involve the values of $f_{e}(v)$ and the derivatives $\frac{d f_{e}}{d x}(v)$ calculated at the vertices. We list some commonly used vertex conditions below.

\footnotetext{
${ }^{2}$ This is a consequence of Barra-Gaspard ergodicity of quantum graphs: informally, what happens once for one choice of lengths will happen with finite frequency for almost all choices of lengths. For more precise statements, see $3,5,11,16$
} 
- Dirichlet condition at a vertex $v \in \mathcal{V}$ requires that the function $f$ vanishes at the vertex,

$$
f(v)=0 .
$$

This is an example of a decoupling condition. Namely, if the Dirichlet condition is imposed at a vertex of degree $d>1$, it is equivalent to disconnecting the edges incident to the vertex and imposing Dirichlet conditions at the resulting $d$ vertices of degree 1 .

- $\delta$-type condition at a vertex $v \in \mathcal{V}$ requires the function to be continuous at $v$ in addition to the condition

$$
\sum_{e \in \mathcal{E}_{v}} \frac{d f_{e}}{d x}(v)=\gamma_{v} f(v), \quad \gamma_{v} \in \mathbb{R}
$$

where $\frac{d f_{e}}{d x}(v)$ is the derivative of the function $f_{e}$ taken in the direction into the edge. We note that the value $f(v)$ is well-defined because of the assumed continuity. The real parameter $\gamma_{v}$ is called the vertex coupling constant. The special case of the $\delta$-type condition with $\gamma_{v}=0$ is the Neumann-Kirchhoff (NK) or "standard" condition. The Dirichlet condition defined above can be naturally interpreted as $\gamma_{v}=+\infty$.

- quasi-NK or magnetic condition at a vertex $v \in \mathcal{V}$ : Assume that the degree of the vertex $v$ is $d_{v}, \mathcal{E}_{v}=\left\{1, \ldots, d_{v}\right\}$ and we are given $d_{v}$ unit complex scalars $z_{1}, \ldots, z_{d_{v}} \in \mathbb{S}^{1}$. We impose the following two conditions:

$$
\left\{\begin{array}{l}
z_{1} f_{1}(v)=z_{2} f_{2}(v)=\ldots=z_{d_{v}} f_{d_{v}}(v) \\
\sum_{j=1}^{d_{v}} z_{j} \frac{d f_{j}}{d x}(v)=0
\end{array}\right.
$$

Of course, the NK condition is a special case of (3) when all $z_{j}$ are equal.

If every vertex of the graph $\mathcal{G}$ is equipped with one of the above conditions, the operator $\mathcal{H}$ is self-adjoint (see [10, Theorem 1.4.4] and references therein). The last set of conditions allow one to introduce magnetic field on the graph without modifying the operator (see [25] and also [32,33] for more recent appearances). They also arise as a result of Floquet-Bloch reduction reviewed in the next section.

3.2. Floquet-Bloch reduction. Let us now return to our periodic graph $X$. Recall that the $\delta$-type conditions are imposed at all vertices of $X$ and hence the operator $-\Delta_{X}$ is self-adjoint. A standard Floquet-Bloch reduction (see e.g., [10, 26, 27|) allows us to reduce the consideration of the spectrum of $-\Delta_{X}$ to a family of spectral problems on a compact quantum graph (a fundamental domain). More precisely, denote by $g_{1}, g_{2}, g_{3}$ some choice of generators of the shift lattice $\mathbb{Z}^{3}$. For each $\mathbf{k}=\left(k_{1}, k_{2}, k_{3}\right) \in(-\pi, \pi]^{3}=: \mathbb{T}^{3}$, let $-\Delta_{X}^{(\mathbf{k})}$ be the Laplacian that acts on the domain consisting of functions $u \in H_{l o c}^{2}(X)$ that satisfy the $\delta$-type conditions at vertices along with the following Floquet conditions,

$$
u_{g_{1} e}(x)=e^{i k_{1}} u_{e}(x), \quad u_{g_{2} e}(x)=e^{i k_{2}} u_{e}(x), \quad u_{g_{3} e}(x)=e^{i k_{3}} u_{e}(x),
$$


for all $x \in X$ and $n=\left(n_{1}, n_{2}, n_{3}\right) \in \mathbb{Z}^{3}$. Then $-\Delta_{X}$ is the direct integral of $-\Delta_{X}^{(\mathbf{k})}$ and therefore,

$$
\sigma\left(-\Delta_{X}\right)=\bigcup_{\mathbf{k} \in \mathbb{T}^{3}} \sigma\left(-\Delta_{X}^{(\mathbf{k})}\right)
$$

The operator $-\Delta_{X}^{(\mathbf{k})}$ has discrete spectrum $\sigma\left(-\Delta_{X}^{(\mathbf{k})}\right)=\left\{\lambda_{j}(\mathbf{k})\right\}_{j=1}^{\infty}$ where we assume that $\lambda_{j}$ is increasing in $j$, see (1). The dispersion relation of the operator $-\Delta_{X}$ is the multivalued function $\mathbf{k} \mapsto\left\{\hat{\lambda}_{j}(\mathbf{k})\right\}$ and the spectrum of $-\Delta_{X}$ is the range of the dispersion relation for quasimomentum $\mathbf{k}$ in $\mathbb{T}^{3}$. Hence, it suffices to focus on solving the eigenvalue problems $-\Delta_{X}^{(\mathbf{k})} u=\lambda u$ where $\lambda \in \mathbb{R}$ for $u$ in the domain of $-\Delta_{X}^{(\mathbf{k})}$. This problem is unitarily equivalent to the eigenvalue problem on the compact graph $\Gamma$,

$$
-\frac{d^{2}}{d x^{2}} u=\lambda u, \quad \lambda \in \mathbb{R}
$$

where $u$ satisfies the respective vertex conditions at the vertices $A$ and $C$ and the quasi-NK conditions at the vertex $B$ :

$$
\left\{\begin{array}{l}
e^{i k_{1}} u_{1}(B)=e^{i k_{2}} u_{2}(B)=e^{i k_{3}} u_{3}(B)=u_{4}(B) \\
e^{i k_{1}} u_{1}^{\prime}(B)+e^{i k_{2}} u_{2}^{\prime}(B)+e^{i k_{3}} u_{3}^{\prime}(B)+u_{4}^{\prime}(B)=\gamma_{B} u(B),
\end{array}\right.
$$

where $\gamma_{B}$ is taken to be 0 for the graph $\Gamma_{2}$ and $u_{j}$ are the restrictions of the function $u$ to the edges $e_{j}$. We will use the notation $\Gamma_{1}^{\mathbf{k}}$ and $\Gamma_{2}^{\mathbf{k}}$ (or $\Gamma^{\mathbf{k}}$ if the distinction between the two graphs is irrelevant) to denote the eigenvalue problem with condition (7) at the vertex $B$.

From now on, we shall emphasize the vertex conditions pictorially by replacing the names of the vertices by their corresponding boundary conditions, see Fig. 5. We will use $\gamma_{A}, \mathbf{N K}, \mathbf{D}$ and $\mathbf{Q}_{\mathbf{k}, \gamma_{\mathbf{B}}}$ to indicate the $\delta$-type, Neumann-Kirchhoff, Dirichlet and quasi-NK vertex conditions respectively. We will also occasionally use this convention in the text, e.g., the vertex $B$ in the above graph $\Gamma^{\mathbf{k}}$ will be mentioned as the $\mathbf{Q}_{\mathbf{k}^{-}}$ vertex. Finally, we will use the symbol $\lambda_{j}\left(\Gamma^{\mathbf{k}}\right)$ for the $j^{t h}$-eigenvalue of the quantum graph $\Gamma^{\mathbf{k}}$. In particular, we have

$$
\sigma\left(-\Delta_{X}\right)=\bigcup_{j \geq 1, k \in \mathbb{T}^{3}}\left\{\lambda_{j}\left(\Gamma^{\mathbf{k}}\right)\right\}
$$
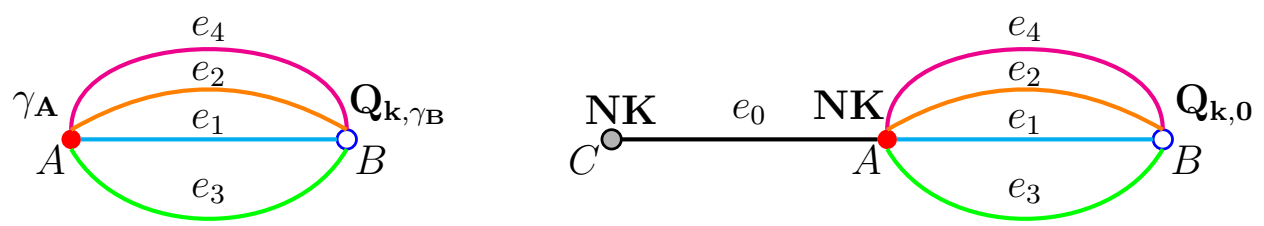

Figure 5. The quantum graphs $\Gamma_{1}^{\mathbf{k}}$ (left) and $\Gamma_{2}^{\mathbf{k}}$ (right) and their vertex conditions. In the figures, the types of the boundary conditions are bold letters while the labels of the vertices are regular letters. 
3.3. Eigenvalue comparison under some surgery transformations. In this section we list some eigenvalue comparison results that will be useful to prove the existence of a gap in the dispersion relation in Theorem 2.1(a).

The following interlacing inequality is often useful when variation of a coupling constant is used to interpolate between different $\delta$-type conditions and also the Dirichlet condition (which is interpreted as the $\delta$-type condition with coupling $+\infty$ ).

Theorem 3.1 (A special case of [8, Theorem 3.4]). If the graph $\widehat{G}$ is obtained from $G$ by changing the coefficient of the $\delta$-type condition at a single vertex $v$ from $\gamma_{v}$ to $\widehat{\gamma}_{v} \in\left(\gamma_{v}, \infty\right]$. Then their eigenvalues satisfy the interlacing inequalities

$$
\lambda_{k}(G) \leq \lambda_{k}(\widehat{G}) \leq \lambda_{k+1}(G) \leq \lambda_{k+1}(\widehat{G}), \quad k \geq 1 .
$$

If a given value $\Lambda$ has multiplicities $m$ and $\widetilde{m}$ in the spectra of $G$ and $\widehat{G}$ respectively, then the $\Lambda$-eigenspaces of $G$ and $\widehat{G}$ intersect along a subspace of dimension $\min (m, \widetilde{m})$. Note that by $(9), \widetilde{m}$ must be equal to $m-1, m$ or $m+1$.

For simplicity, from now on, if the graph $G_{1}$ is obtained from $G_{2}$ by changing the $\delta$-type conditions to Dirichlet conditions at a single vertex, we will say that $G_{1}$ is a rank one Dirichlet perturbation of the graph $G_{2}$.

We now consider the effect on the eigenvalue of the enlargement of a graph, which is realized by attaching a subgraph at a designated vertex. The following theorem is quoted in the narrowest form that is sufficient for our needs.

Theorem 3.2 (A special case of [8, Theorem 3.10]). Suppose that $\widehat{G}$ is formed from graphs $G$ and $H$ by identifying or "gluing" two Neumann-Kirchhoff vertices $v_{0} \in G$ and $w_{0} \in H$. If $\lambda_{1}(H)<\lambda_{1}(G)$ and the eigenvalue $\lambda_{1}(G)$ has an eigenfunction which does not vanish at $v_{0}$ then $\lambda_{1}(\widehat{G})<\lambda_{1}(G)$.

3.4. Topology of moduli spaces of polygons. Given $n$ positive real numbers $\left\{a_{j}\right\}$ one can ask what is the topology of the space of all planar polygons whose side lengths are $\left\{a_{j}\right\}$. Two polygons are identified if they can be mapped into each other by a composition of rotation and translation. The resulting spaces may not be smooth and their full classification is surprisingly rich, see [17] and references therein. These spaces make an appearance in our question as the degenerate curves on which the dispersion relation has an extremum.

For our example we will only require the following simple lemma (which follows from the results of [17]) addressing the topology of the set of quadrangles with given four edge lengths, see Figure 6.

Lemma 3.3. The curve $\mu$ of solutions $\mathbf{k}=\left(k_{1}, k_{2}, k_{3}\right) \in \mathbb{T}^{3}$ of

$$
\sum_{1 \leq j \leq 3} e^{i k_{j}} a_{j}+a_{4}=0
$$

is an algebraic curve of co-dimension 2 if and only if

$$
a_{m}<\sum_{j \neq m} a_{j}
$$

for every $m=1, \ldots, 4$. 


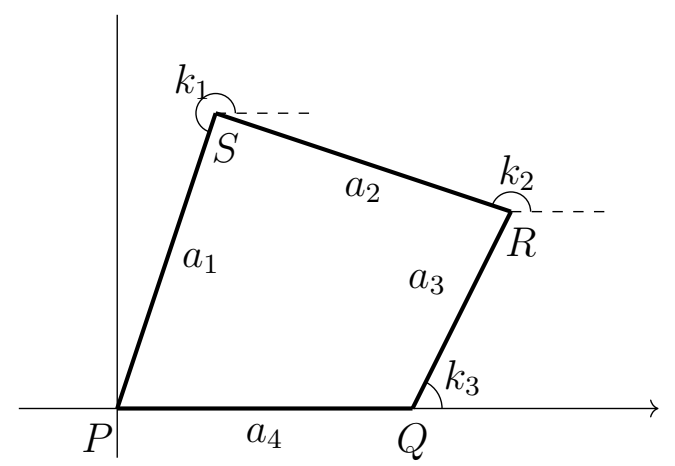

FIGURE 6. Quadrangle corresponding to equation (10).
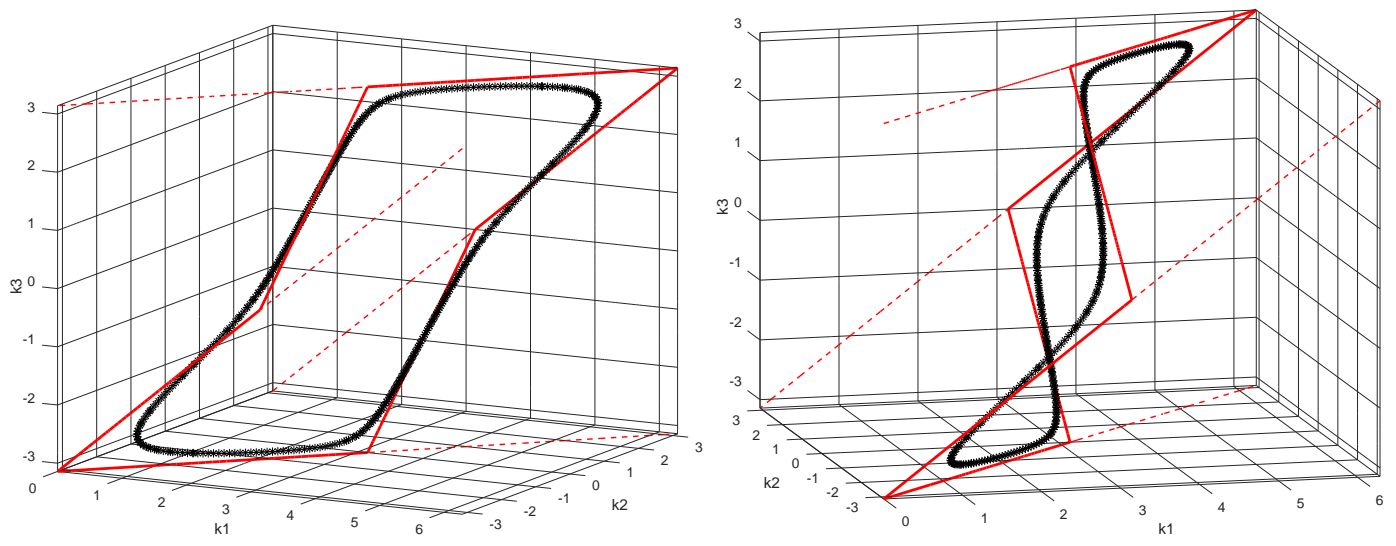

Figure 7. The set of roots of $(10)$ for two choices of $\left\{a_{j}\right\}$; two views of the same plot are shown. The ranges are adjusted to $k_{1} \in(0,2 \pi]$ and $k_{2}, k_{3} \in(-\pi, \pi]$ for a smoother plot. Straight red lines correspond to $a_{j}=1$ for all $j$; Black stars (appear as a thick fuzzy line) are produced using $a_{1}=1.1, a_{2}=0.95, a_{3}=0.9$ and $a_{4}=1$.

If there is an $m$ with the inequality reversed, the set of solutions $\mu$ is empty. If there is an $m$ with inequality turning into equality, the set of solutions is a single point.

The topology of $\mu$ in this particular case has been described, for example, in [19, Sec 12]. The curve is smooth unless there is a linear combination of $\left\{a_{j}\right\}$ with coefficients \pm 1 that is equal to zero. If the curve $\mu$ is smooth it is either a circle or a disjoint union of two circles. The non-generic cases when $\mu$ is not a smooth manifold are of the following types: two circles intersecting at a point, two circles intersecting at two points and three circles with one intersection among each pair.

The latter case arises when all $a_{j}$ are equal. It is shown in red solid line in Figure 7 . Note that the plot is on a torus, therefore each pair of parallel lines is actually a single line forming a circle. A smooth curve $\mu$ for a generic choice of $a_{j} \approx 1$ is also shown. 


\section{Proof of the MAIN Result}

In this section, we present the details of the proof of Theorem 2.1. Without loss of generality, for the graph $\Gamma_{1}$ we will make the assumption

$$
\gamma_{A}<\gamma_{B}
$$

Starting with the graph $\Gamma_{1}$, we introduce two of its modifications. The graph $\Gamma_{1}^{A}$ is obtained by changing the condition at the vertex $A$ to Dirichlet; the graph $\Gamma_{1}^{B}$ is obtained similarly by placing a Dirichlet condition at the vertex $B$. Remembering that a Dirichlet condition is decoupling, we can picture the result as shown in Fig. 8 ,
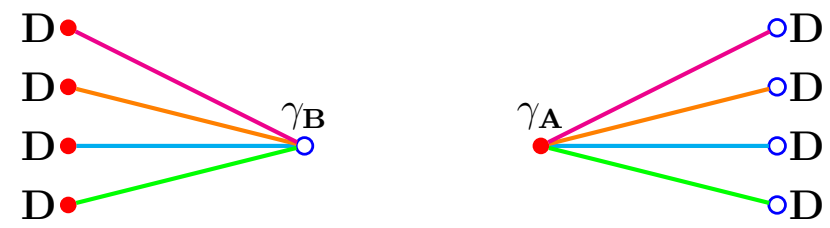

Figure 8. The two "star" graphs $\Gamma_{1}^{A}$ (left) and $\Gamma_{1}^{B}$ (right) after disconnecting the corresponding Dirichlet vertices $(\mathbf{D})$.

By placing Dirichlet conditions at vertices $A$ or $B$ of the graph $\Gamma_{2}$, we analogously construct the two graphs $\Gamma_{2}^{A}$ and $\Gamma_{2}^{B}$. We remark that the graph $\Gamma_{2}^{A}$ has two connected components, see Fig. 9 and Fig. 10 . Using the tools introduced in Section 3.3 we establish the following comparison result, which compares the first eigenvalue $\Gamma_{j}^{A}$ with the first eigenvalue of $\Gamma_{j}^{B}$, where $j$ is either 1 or 2 .

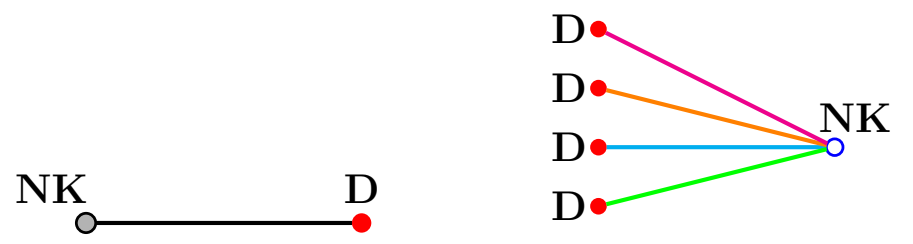

Figure 9. The quantum graphs $\Gamma_{2}^{A, 1}$ (left) and $\Gamma_{2}^{A, 2}$ after disconnecting from the Dirichlet vertex of their union $\Gamma_{2}^{A}$.

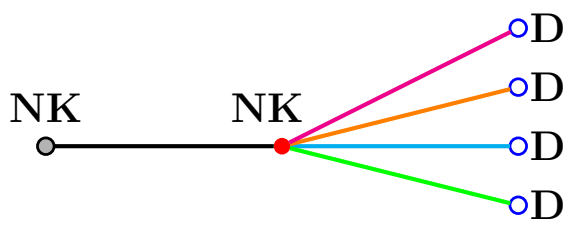

Figure 10. The quantum graph $\Gamma_{2}^{B}$.

Lemma 4.1. The first eigenvalue of $\Gamma^{B}$ is always strictly less than than the first eigenvalue of $\Gamma^{A}$,

$$
\lambda_{1}\left(\Gamma^{B}\right)<\lambda_{1}\left(\Gamma^{A}\right)
$$


Proof. The graphs $\Gamma_{1}^{B}$ and $\Gamma_{1}^{A}$ differ only in the coefficient of the $\delta$-type condition at the vertex of degree 4 (we are in the situation of pure Laplacian, with no potential). Since the coefficient of $\Gamma_{1}^{B}$ (which is $\gamma_{A}$ ) is smaller than the coefficient of $\Gamma_{1}^{A}$, see equation (12), we immediately get from Theorem 3.1 that $\lambda_{1}\left(\Gamma_{1}^{B}\right) \leq \lambda_{1}\left(\Gamma_{1}^{A}\right)$. The case of equality is excluded because the ground state must be non-zero on the vertex of degree 4 which means it cannot satisfy $\delta$-type conditions with two different constants (hence it cannot be a common eigenfunction).

For the graph $\Gamma_{2}$ we establish two inequalities, $\lambda_{1}\left(\Gamma_{2}^{B}\right)<\lambda_{1}\left(\Gamma_{2}^{A, 1}\right)$ and $\lambda_{1}\left(\Gamma_{2}^{B}\right)<$ $\lambda_{1}\left(\Gamma_{2}^{A, 2}\right)$. The first follows by changing the condition at vertex $A$ of the graph $\Gamma_{2}^{B}$ from NK to Dirichlet: the eigenvalue strictly increases (since the eigenfunction of $\Gamma_{2}^{B}$ is non-zero at $A$ ) and the graph decouples into several disjoint parts one of which coincides with $\Gamma_{2}^{A, 1}$.

To prove the second inequality, we start with $\lambda_{1}\left(\Gamma_{2}^{A, 2}\right)>0$ whose eigenfunction does not vanish on vertex $B$, and attach to $B$ a Neumann interval of length $\ell_{0}$ whose first eigenvalue is $0<\lambda_{1}\left(\Gamma_{2}^{A, 2}\right)$. The strict inequality follows from Theorem 3.2 .

In our terminology, the graphs $\Gamma^{A}$ and $\Gamma^{B}$ are the rank one Dirichlet perturbations of the corresponding graph $\Gamma$. The next important observation is that they are also, in fact, the rank one Dirichlet perturbations of the corresponding graph $\Gamma^{\mathbf{k}}$ for any k.

Lemma 4.2. The rank one Dirichlet perturbation of the graph $\Gamma^{\mathbf{k}}$ at the vertex $A$ (corresp. B) is unitarily equivalent to $\Gamma^{A}$ (corresp. $\Gamma^{B}$ ) for any $\mathbf{k} \in \mathbb{T}^{3}$.

Proof. Since the Dirichlet perturbation is decoupling, the resulting graphs have no cycles and therefore any quasi-momenta can be removed by a gauge transform, see [10, Thm 2.6.1]. To put it another way, replacing the vertex condition (7) at $B$ with Dirichlet removes all dependence on the quasi-momenta k. Similarly, the quasi-NK conditions could be equivalently imposed at the vertex $A$, where replacing them with Dirichlet also removes all dependence on $\mathbf{k}$.

Lemma 4.3. The first eigenvalue $\lambda_{1}$ of $-\Delta$ on $\Gamma^{B}$ is simple. If $\ell_{1}=\ell_{2}=\ell_{3}=\ell_{4}$, the eigenfunction corresponding to $\lambda_{1}$ is identical on these four edges, $\phi_{1} \equiv \phi_{2} \equiv \phi_{3} \equiv \phi_{4}$, and non-zero except at $B$.

Proof. The proof is identical for $\Gamma_{1}^{B}$ and $\Gamma_{2}^{B}$. Simplicity of the eigenvalue follows from general variational principles $[32]$ (or can be deduced from the secular equation for the corresponding graphs, see also the proof of Proposition 4.4 below). The first eigenfunction is known to be positive, except where a Dirichlet condition is enforced, for a large family of vertex conditions 32]. Symmetry can be deduced by, for example, restricting $-\Delta$ to the symmetric subspace of the operator's domain [4], observing that the first eigenfunction of the restricted operator is positive and concluding that it corresponds to a positive eigenfunction of the full operator and therefore must be the ground state.

Proof of Theorem 2.1. Since by Lemma 4.2 $\Gamma^{A}$ and $\Gamma^{B}$ are obtained by a rank-1 Dirichlet perturbation from the quantum graph $\Gamma^{\mathbf{k}}$ for any $\mathbf{k}$, Theorem 3.1 yields the 
inequalities

$$
\lambda_{1}\left(\Gamma^{\mathbf{k}}\right) \leq \lambda_{1}\left(\Gamma^{B}\right) \leq \lambda_{2}\left(\Gamma^{\mathbf{k}}\right),
$$

and

$$
\lambda_{1}\left(\Gamma^{\mathbf{k}}\right) \leq \lambda_{1}\left(\Gamma^{A}\right) \leq \lambda_{2}\left(\Gamma^{\mathbf{k}}\right),
$$

which hold of all $\mathbf{k} \in \mathbb{T}^{3}$. Adding the result of Lemma 4.1 , we get

$$
\lambda_{1}\left(\Gamma^{\mathbf{k}}\right) \leq \lambda_{1}\left(\Gamma^{B}\right)<\lambda_{1}\left(\Gamma^{A}\right) \leq \lambda_{2}\left(\Gamma^{\mathbf{k}}\right),
$$

obtaining part (a) of Theorem 2.1.

We will now show that the first inequality in (16) turns into equality

$$
\lambda_{1}\left(\Gamma^{\mathbf{k}}\right)=\lambda_{1}\left(\Gamma^{B}\right)
$$

for $k$ in a one-dimensional curve $\gamma$ in $\mathbb{T}^{3}$.

Let $\varphi$ be the $\lambda_{1}\left(\Gamma^{B}\right)$-eigenfunction of $\Gamma^{B}$. By Theorem 3.1, equality (17) holds if and only if $\varphi$ is also an eigenfunction of $\Gamma^{\mathrm{k}}$. We denote by $\varphi_{j}$ the restriction of $\varphi$ on $e_{j}$ for $0 \leq j \leq 4$. Obviously, $\varphi$ satisfies the first condition in (7) at the vertex $B$. Therefore, equality (17) holds if and only if $\mathbf{k}=\left(k_{1}, k_{2}, k_{3}\right) \in[-\pi, \pi)^{3}$ is such that

$$
\sum_{1 \leq j \leq 3} e^{i k_{j}} \varphi_{j}^{\prime}(B)+\varphi_{4}^{\prime}(B)=0
$$

By Lemma 3.3. the set of solutions of $(18)$ is a non-trivial algebraic curve of codimension 2 if

$$
2 \max _{j}\left|\varphi_{j}^{\prime}(B)\right|<\sum_{1 \leq j \leq 4}\left|\varphi_{j}^{\prime}(B)\right| .
$$

If the lengths $\ell_{j}(1 \leq j \leq 4)$ are approximately equal then (by eigenfunction continuity and Lemma 4.3$)$ all $\left|\varphi_{j}^{\prime}(B)\right|$ are approximately equal and condition (19) is satisfied. This completes the proof of part (b).

Finally, the robustness of the degenerate gap edge under a small perturbation of edge lengths or edge potentials follows directly from continuity of eigenvalue and eigenfunction data 9,31 and the fact that conditions for the degenerate gap edge are inequalities (13) and (19).

With a little extra effort we can provide a quantitative condition on the lengths $\ell_{j}$ to ensure the validity of the quadrangle inequalities 190 whenever all of the derivatives $\varphi_{1}^{\prime}(B), \ldots, \varphi_{4}^{\prime}(B)$ are not zero.

Proposition 4.4. Let $\rho_{0}$ be the unique solution in $(2,3)$ to the equation

$$
\rho^{2}-\frac{\rho^{3}}{3}=\frac{\pi^{2}}{24}
$$

and assume further that

$$
\min \left\{\left(\rho_{0} \cdot \min _{1 \leq j \leq 4} \ell_{j}\right), \ell_{0}\right\} \geq \max _{1 \leq j \leq 4} \ell_{j}
$$

Then $2 \cdot\left|\varphi_{j}^{\prime}(B)\right|<\left|\varphi_{1}^{\prime}(B)\right|+\left|\varphi_{2}^{\prime}(B)\right|+\left|\varphi_{3}^{\prime}(B)\right|+\left|\varphi_{4}^{\prime}(B)\right|$ for each $1 \leq j \leq 4$. As a consequence, the same conclusion in part (b) of Theorem 2.1 holds. 
Proof. Without loss of generality, assume that $\ell_{4} \geq \ell_{3} \geq \ell_{2} \geq \ell_{1}$. On the edge $e_{j}$ where $1 \leq j \leq 4$, we write $\varphi_{j}(x)=\alpha_{j} \sin (\beta x)$, where $0 \leq x \leq \ell_{j}, \alpha_{j} \in \mathbb{R}$ and $\beta=\left(\lambda_{1}\left(\Gamma^{B}\right)\right)^{1 / 2}$. Here we identify the vertex $B$ as $x=0$ on each edge $e_{j}$. Observe that

$$
0<\beta=\lambda_{1}\left(\Gamma^{B}\right)^{1 / 2} \leq \min _{1 \leq j \leq 4}\left\{\frac{\pi}{2 \ell_{0}}, \frac{\pi}{\ell_{j}}\right\}=\frac{\pi}{2 \ell_{0}}
$$

This implies that $\beta \ell_{j} \in(0, \pi / 2]$ for each $j$. So $\min _{1 \leq j \leq 4}\left|\sin \left(\beta \ell_{j}\right)\right|=\sin \left(\beta \ell_{1}\right)$. Moreover, from the fact that $\varphi_{j}\left(\ell_{j}\right) \neq 0$ and the continuity of $\varphi$ at the vertex $A$, we have

$$
\beta^{-1} \varphi_{j}^{\prime}(B)=\alpha_{j}=\alpha_{4} \cdot \frac{\sin \left(\beta \ell_{4}\right)}{\sin \left(\beta \ell_{j}\right)}
$$

Therefore, it is enough to show

$$
\sin \left(\beta \ell_{1}\right) \cdot \sum_{i=2}^{4} \frac{1}{\sin \left(\beta \ell_{i}\right)}>1
$$

Put $\rho:=\frac{\ell_{4}}{\ell_{1}} \in\left[1, \rho_{0}\right]$ then we get

$$
1-\frac{\pi^{2}}{24 \rho^{2}}>\frac{\rho}{3}
$$

From $20, \beta \ell_{1}<\frac{\pi \ell_{1}}{2 \ell_{0}} \leq \frac{\pi}{2 \rho}$ and so it implies

$$
1-\frac{\left(\beta \ell_{1}\right)^{2}}{6}>\frac{\ell_{4}}{3 \ell_{1}}
$$

Since $\sin \left(\beta \ell_{1}\right) \geq \beta \ell_{1}-\left(\beta \ell_{1}\right)^{3} / 6$ and $\sin \left(\beta \ell_{j}\right) \leq\left(\beta \ell_{4}\right), 21$ follows from 22 .

\section{Discussion}

Our Theorem 2.1 provides a quantum graph counterexample to the mentioned conjecture at the beginning of the paper, about the genericity of non-degenerate spectral edges in spectra of $\mathbb{Z}^{d}$-periodic quantum graphs, where $d>2$. Note that this construction can also be modified to provide an example of a $\mathbb{Z}^{d}$-discrete graph whose dispersion relation of the discrete Laplacian operator contains a degenerate band edge. Indeed, let $\Gamma_{d}$ be the graph with two vertices such that there are exactly $d+1$ - edges between them and therefore, its maximal abelian covering $X_{d}$ is a $d$-dimensional topological diamond. One can write down explicitly the dispersion relation of $X_{d}$ and then proceed a similar calculation as in [18 to derive the degeneracy of the extrema of the band functions.

Our construction of the graphs $\Gamma$ required that the dimension of the dual torus of quasimomenta $k$ be of dimension at least three. The same method and proof will still work if we increase the number of edges connecting the two vertices $A$ and $B$ (at least four edges). In fact, the entire mechanism of the proof is extremely robust: two rank-one perturbations that reduce the number of cycles by 3 or more help create the gaps between conductivity bands, while a continuum of solutions to an equation similar to (18) will make the band edge degenerate. The degeneracy curve thus still 
has a natural interpretation as the set of possible $(n-1)$-tuples of angles in a planar $n$-gon with the given edge lengths; here $n$ is the number of cycles broken by the rank one perturbation. For $n>3$ the topology of such objects becomes increasingly

complicated. Their homology groups were studied by many authors, see [17] and references therein.

A heuristic reason for the degeneracy may be put forward using the classical idea of Wigner and von Neumann: a family of complex Hermitian matrices depending on 3 parameters is expected to have isolated point degeneracies (where a pair of eigenvalues meet). This is what the eigenvalues want to do here, but there are hard bounds (14) and (15) from the rank one perturbations, so the eigenvalues instead accumulate at the bound.

\section{Acknowledgments}

The work of the first author was partially supported by NSF DMS-1815075 grant and the work of the second author was partially supported by the AMS-Simons Travel grant. Both authors express their gratitude to Peter Kuchment for introducing them to this exciting topic and to Lior Alon and Ram Band for many deep discussions. We thank an anonymous referee for several improving suggestions.

\section{REFERENCES}

[1] N. W. Ashcroft and N. D. Mermin, Solid State Physics, Holt, Rinehart and Winston, New York-London, 1976.

[2] J. Baez, Topological Crystals, 2016. preprint arXiv:1607.07748.

[3] R. Band and G. Berkolaiko, Universality of the Momentum Band Density of Periodic Networks, Phys. Rev. Lett. 111 (2013), 130404, DOI 10.1103/PhysRevLett.111.130404.

[4] R. Band, G. Berkolaiko, C. H. Joyner, and W. Liu, Quotients of finite-dimensional operators by symmetry representations, 2017. preprint arXiv:1711.00918.

[5] F. Barra and P. Gaspard, On the level spacing distribution in quantum graphs, J. Statist. Phys. 101 (2000), no. 1-2, 283-319, DOI 10.1023/A:1026495012522.

[6] G. Berkolaiko, An elementary introduction to quantum graphs, Geometric and computational spectral theory, 2017, pp. 41-72, DOI 10.1090/conm/700/14182.

[7] G. Berkolaiko and A. Comech, Symmetry and Dirac points in graphene spectrum, J. Spectr. Theory 8 (2018), no. 3, 1099-1147, DOI 10.4171/JST/223. preprint arXiv:1412.8096.

[8] G. Berkolaiko, J. B. Kennedy, P. Kurasov, and D. Mugnolo, Surgery principles for the spectral analysis of quantum graphs, Trans. Amer. Math. Soc (2019).

[9] G. Berkolaiko and P. Kuchment, Dependence of the spectrum of a quantum graph on vertex conditions and edge lengths, Spectral Geometry, 2012. preprint arXiv:1008.0369.

[10] G. Berkolaiko and P. Kuchment, Introduction to quantum graphs, Mathematical Surveys and Monographs, vol. 186, American Mathematical Society, Providence, RI, 2013.

[11] G. Berkolaiko and B. Winn, Relationship between scattering matrix and spectrum of quantum graphs, Trans. Amer. Math. Soc. 362 (2010), no. 12, 6261-6277, DOI 10.1090/S0002-9947-201004897-4.

[12] M. Sh. Birman, On the homogenization for periodic operators in a neighborhood of an edge of an internal gap, Algebra i Analiz 15 (2003), no. 4, 61-71.

[13] M. Sh. Birman and T. A. Suslina, Threshold effects near the lower edge of the spectrum for periodic differential operators of mathematical physics, Systems, approximation, singular integral operators, and related topics (Bordeaux, 2000), 2001, pp. 71-107. 
[14] _ Homogenization of a multidimensional periodic elliptic operator in a neighborhood of an edge of an inner gap, Zap. Nauchn. Sem. S.-Peterburg. Otdel. Mat. Inst. Steklov. (POMI) 318 (2004), no. Kraev. Zadachi Mat. Fiz. i Smezh. Vopr. Teor. Funkts. 36 [35], 60-74, 309.

[15] N. Do, P. Kuchment, and F. Sottile, Generic properties of dispersion relations for discrete periodic operators (2019). preprint arXiv:1910.06472

[16] P. Exner and O. Turek, Periodic quantum graphs from the Bethe-Sommerfeld perspective, J. Phys. A 50 (2017), no. 45, 455201, 32, DOI 10.1088/1751-8121/aa8d8d.

[17] M. Farber and D. Schütz, Homology of planar polygon spaces, Geom. Dedicata 125 (2007), $75-92$.

[18] N. Filonov and I. Kachkovskiy, On the structure of band edges of 2-dimensional periodic elliptic operators, Acta Math. 221 (2018), no. 1, 59-80.

[19] M. Kapovich and J. Millson, On the moduli space of polygons in the Euclidean plane, J. Differential Geom. 42 (1995), no. 1, 133-164.

[20] M. Kha, Green's function asymptotics of periodic elliptic operators on abelian coverings of compact manifolds, J. Funct. Anal. 274 (2018), no. 2, 341-387.

[21] M. Kha, P. Kuchment, and A. Raich, Green's function asymptotics near the internal edges of spectra of periodic elliptic operators. Spectral gap interior, J. Spectr. Theory 7 (2017), no. 4, $1171-1233$.

[22] W. Kirsch and B. Simon, Comparison theorems for the gap of Schrödinger operators, J. Funct. Anal. 75 (1987), no. 2, 396-410.

[23] F. Klopp and J. Ralston, Endpoints of the spectrum of periodic operators are generically simple, Methods Appl. Anal. 7 (2000), no. 3, 459-463.

[24] T. Kobayashi, K. Ono, and T. Sunada, Periodic Schrödinger operators on a manifold, Forum Math. 1 (1989), no. 1, 69-79.

[25] V. a. S. Kostrykin R., Quantum wires with magnetic fluxes, Comm. Math. Phys. 237 (2003), no. 1-2, 161-179.

[26] P. Kuchment, Floquet theory for partial differential equations, Operator Theory: Advances and Applications, vol. 60, Birkhäuser Verlag, Basel, 1993.

[27] _ An overview of periodic elliptic operators, Bulletin (New Series) of the American Mathematical Society 53 (July 2016), no. 3, 343-414.

[28] P. Kuchment and Y. Pinchover, Integral representations and Liouville theorems for solutions of periodic elliptic equations, J. Funct. Anal. 181 (2001), no. 2, 402-446.

[29] L Liouville theorems and spectral edge behavior on abelian coverings of compact manifolds, Trans. Amer. Math. Soc. 359 (2007), no. 12, 5777-5815.

[30] P. Kuchment and A. Raich, Green's function asymptotics near the internal edges of spectra of periodic elliptic operators. Spectral edge case, Math. Nachr. 285 (2012), no. 14-15, 1880-1894.

[31] P. Kuchment and J. Zhao, Analyticity of the spectrum and Dirichlet-to-Neumann operator technique for quantum graphs, J. Math. Phys. 60 (2019), no. 9, 093502, 8, DOI 10.1063/1.5110193.

[32] P. Kurasov, On the ground state for quantum graphs, Lett. Math. Phys. 109 (2019), no. 11, 2491-2512, DOI 10.1007/s11005-019-01192-w.

[33] D. Mugnolo, Semigroup methods for evolution equations on networks, Understanding Complex Systems, Springer, Cham, 2014.

[34] L. Parnovski and R. Shterenberg, Perturbation theory for spectral gap edges of $2 D$ periodic Schrödinger operators, J. Funct. Anal. 273 (2017), no. 1, 444-470.

[35] T. Sunada, Topological crystallography, Surveys and Tutorials in the Applied Mathematical Sciences, vol. 6, Springer, Tokyo, 2013. With a view towards discrete geometric analysis. 
G.B., Department of Mathematics, Texas A\&M University, College Station, TX 77843-3368, USA

E-mail address: Gregory.Berkolaiko@math.tamu.edu

M.K., Department of Mathematics, The University of Arizona, Tucson, AZ 857210089, USA

E-mail address: minhkha@math.arizona.edu 